\title{
Numerical modeling of dielectric barrier discharge actuators based on the properties of low-frequency plasmons
}

\section{Soltani Tehrani}

Amirkabir University of Technology

G. R. Abdizadeh ( $\nabla$ g.abdizadeh@aut.ac.ir)

Amirkabir University of Technology

S. Noori

Amirkabir University of Technology

\section{Research Article}

Keywords:

Posted Date: February 22nd, 2022

DOI: https://doi.org/10.21203/rs.3.rs-1013871/v1

License: (c) (i) This work is licensed under a Creative Commons Attribution 4.0 International License.

Read Full License 


\title{
Numerical modeling of dielectric barrier discharge actuators based on the properties of low-frequency plasmons
}

\author{
D.Soltani Tehrani ${ }^{1}$, G.R.Abdizadeh ${ }^{1, *}$, and S.Noori ${ }^{1}$ \\ ${ }^{1}$ Amirkabir University of Technology, Aerospace Engineering Department, Tehran, postcode, Iran \\ *g.abdizadeh@aut.ac.ir
}

\begin{abstract}
Electrohydrodynamic flow control systems have proven to be among the most promising flow control strategies within previous decades. Several methods for efficient evaluation and description of the effect of such systems are indeed available. Yet, due to these systems' critical role in various applications, possible improvements are still investigated. A new phenomenological model is presented for the simulation of the plasma actuators based on the electrodynamic properties of low-frequency plasmons. The model simulates the plasmonic region as a dispersive medium. This dissipated energy is added to the flow by introducing a high-pressure region, calculated in terms of local body force vectors, requiring the distribution of the electric field and the polarization field. The model determines the electric field for the computation of the body force vector based on the Poisson equation and implements the simplified Lorentz model for the polarization field. To fully explore the performance of the presented model, an experiment has been conducted providing a comparison between the observed effect of plasma actuators on the fluid flow with the results predicted by the model. The model is then validated based on the results of other distinct experiments and exempted numerical models, based on the exchanging momentum with the ambient neutrally charged fluid, demonstrating that the model has improved adaptability and self-adjusting capability compared to the available models.
\end{abstract}

\section{Introduction}

Electrohydrodynamic flow control systems have proven to be among the most promising flow control strategies within previous decades. Among these systems, plasma actuators have been confirmed to be effective in a wide variety of applications, including flow control purposes, photonics and optoelectronics, food processing technologies, cancer treatment, and biotechnology ${ }^{1-6}$. Literature shows a strong background, investigating and improving the applicability and effectiveness of different flow control methods in several application fields ${ }^{7-16}$. However, a thorough development and testing process is required to incorporate the resulting systems into actual applications. Numerical simulations have traditionally attempted to give advanced algorithms for designing, simulating, and comprehending complicated flow control systems, as the experimental approach requires several costly and time-consuming trial-and-error iterations. Several methods for efficient evaluation and description of the effect of dielectric barrier discharge (DBD) systems are currently available through the literature. However, due to these systems' critical role in many flow control problems, possible improvements are always worth investigating, and an improved algorithm is always welcome.

There are currently three categories of models for simulating plasma actuators; models based on fundamental principles ${ }^{17,18}$, empirical models ${ }^{19,20}$, and phenomenological models ${ }^{21-25}$. Models in the first category attempt to reproduce the exact physical mechanisms of a plasma actuator, which necessitate the solution of complex transport equations for both charged and neutral species, as well as the Poisson equation for the electric field and the Navier-Stokes equations. These models are of more accuracy while requiring high computational cost and time. The second category attempts to impose an accurate description of the induced body force of plasma actuators in the momentum equations. These models consider developing practical modeling tools for DBD actuators for fast design, control, and optimization purposes. The final category of models uses simplified sets of differential equations, resulting in less computationally demanding simulations while considering the contributing physics with simplifications and maintaining an acceptable level of accuracy. There has been plenty of research investigating the properties of plasma actuators from various aspects over recent years. In the first place, the current work reviews some of the exempted past experimental and numerical research on plasma actuators, then discusses thoughts and fundamentals in order to better understand the underlying physical mechanisms of the actuator's interaction with the flow and to develop a new practical methodology for simulating plasma actuators.

The plasma actuators are comprised of two electrodes separated by a dielectric substance, as shown in Figure 1. The air around the electrodes ionizes weakly when an AC voltage is applied to them. Differences between the phenomenological 
models come into a discussion based on ways of characterizing and then implementing the consequences of this weakly ionized medium, considered the plasmonic medium. Shyy et al. ${ }^{23}$ characterized the exterior flow effects of the plasma actuators as a time-averaged mean body force spread in a triangular area above the embedded electrode. Suzen and Huang ${ }^{24,26}$ proposed a model based on the plasma formulation of Enloe et al. ${ }^{27}$, reducing the Maxwell equations, considering the plasma formation as a quasi-steady process, and ignoring magnetic forces. In the Navier-Stokes equations, the induced body force was introduced as a source term. The charge distribution over the dielectric surface was assumed to have a 1-D Gaussian distribution based on the experimental data ${ }^{28}$. Many improvements to this formulation have been presented in the literature ${ }^{25,29-32}$. Orlov and Corke ${ }^{33,34}$ used a lumped parameter model to simulate the plasma actuator effects. In contrast to these straightforward methodologies, to explain collisional surface DBD actuators at atmospheric pressures, Roy and Gaitonde ${ }^{35,36}$ employed the solution of charge continuity, momentum, electric field, and electric potential distribution. The electric force obtained as a charge and electric field has been employed to compute the flow actuation effect across the literature ${ }^{33-38}$. Table 1 summarizes the features of the previous models in order for a better comparison.

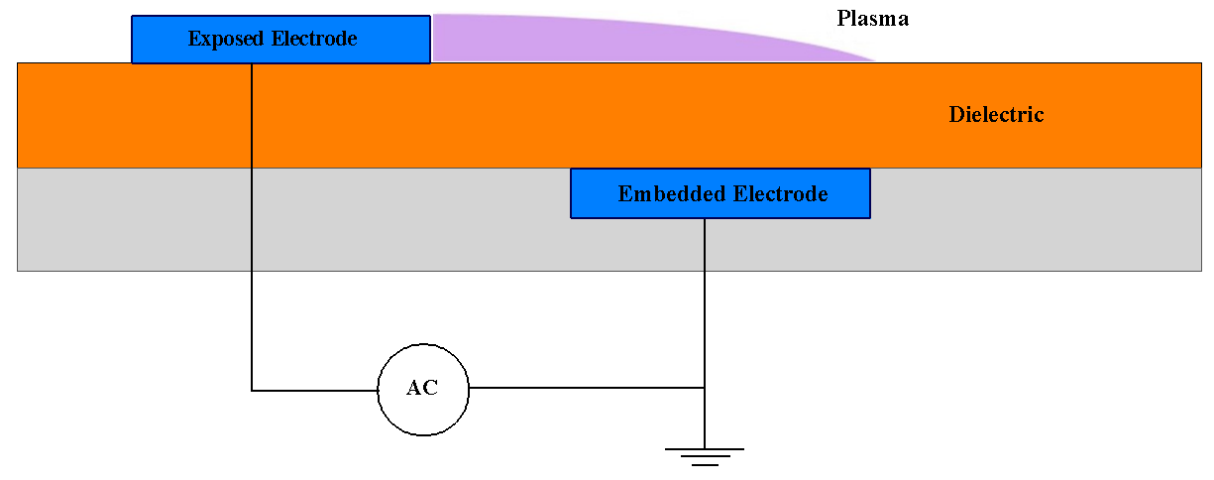

Figure 1. DBD plasma actuator schematic

A literature review reports that much effort has been expended on simulating the plasma actuation effect on fluid flow as accurately as feasible while keeping the computational cost near the optimum. However, the previous works introduce the plasmonic region as a volume charge density distribution, being exposed to an electric field that produces and transfers momentum into the fluid flow. These models require characterizing parameters to be regulated based on the experiments due to changes in the actuator's excitation features or configuration. The concerns appear when new fields of application for these controlling devices face scalability, layout design, and optimization challenges, and the existing models fail to provide enough flexibility.

Despite the fact that computational investigation of the underlying physics of DBD actuators has been extremely difficult, treating the plasmonic region as an excited material based on its frequency responsive properties, rather than predicting a space charge density distribution for the region, leads to a modulable model. We aim to present a new numerical methodology for plasma actuator-based active flow control applications. In this innovative approach, the plasmonic zone is replicated using a practical material model, the Lorentz model ${ }^{39}$. The next section goes into the model's development and implementation in detail. The electrodynamic computations for a single plasma actuator and computations of flow control in quiescent flow using a single plasma actuator are given in the "Results and Discussion" section. Concluding remarks would come in the section titled "Conclusion." The final section titled "Method" provides details of the conducted experiments.

\section{Development of The Computational Model}

A DBD plasma actuator is mounted on the surface, with one electrode exposed to the environment and the other embedded in the dielectric material below the surface (Figure 1). The plasmonic zone is created when high-voltage AC is delivered to the electrodes, which causes the air around them to ionize weakly. Assuming the plasma is quasi-neutral with ions too heavy to respond to electromagnetic field fluctuations, coupling between the electromagnetic response of the medium and the plasma occurs primarily through the electron current density. Due to the wave impedance of the medium in which an electromagnetic 


\begin{tabular}{|c|c|c|}
\hline Reference & Contribution & Main Features \\
\hline Shyy et al. ${ }^{23}$ & $\begin{array}{l}\text { Models the influence of plasma actuators on external } \\
\text { flow as a time-averaged mean body force spread in a } \\
\text { triangular area above the implanted electrode; } \\
\text { linearizes the electric field. }\end{array}$ & $\begin{array}{c}\text { Based on experimental results, uses a } \\
\text { predetermined volume charge density } \\
\text { and plasma volume. }\end{array}$ \\
\hline Suzen et al. ${ }^{24,26}$ & $\begin{array}{l}\text { Calculates the body force by solving the Poisson } \\
\text { equation and then adding the resultant body force to } \\
\text { the Navier-Stokes equations as a source term. }\end{array}$ & $\begin{array}{l}\text { Assumes a Gaussian charge } \\
\text { distribution and introduces maximum } \\
\text { charge density and standard deviation } \\
\text { parameters that will be regulated } \\
\text { based on experimental results. }\end{array}$ \\
\hline Orlov et al. ${ }^{33,34}$ & $\begin{array}{l}\text { To find the actuator body force vector, which is } \\
\text { utilized to govern the amount of energy injected into } \\
\text { the flow, solves a time-dependent charge distribution } \\
\text { equation to provide boundary conditions for the } \\
\text { electric field equation. }\end{array}$ & $\begin{array}{c}\text { No need to make a prior assumption } \\
\text { of charge density boundary condition } \\
\text { while solving; adds an extra equation } \\
\text { to regulate the injected energy into the } \\
\text { fluid flow. }\end{array}$ \\
\hline $\begin{array}{l}\text { Abdollahzedeh et } \\
\text { al. }^{25}\end{array}$ & $\begin{array}{l}\text { The model of Suzen and Huang has been improved; } \\
\text { constructs a simple model for plasma discharge and } \\
\text { its effect on flow, based on scaling the thrust } \\
\text { produced by DBD plasma actuators. To estimate and } \\
\text { simulate the body force distribution created by the } \\
\text { plasma actuator, introduces scales into a basic } \\
\text { phenomenological model. }\end{array}$ & $\begin{array}{c}\text { Adds an extra equation to scale the } \\
\text { thrust of the actuator; properly } \\
\text { forecasts the nonlinear relationship } \\
\text { between thrust produced and applied } \\
\text { voltage. }\end{array}$ \\
\hline Omidi el al. ${ }^{29,30}$ & $\begin{array}{l}\text { Suzen and Huang's model has been improved; adds } \\
\text { an empirical relationship to relate variations in } \\
\text { frequency to the Debye length as one of the } \\
\text { characterizing factors. }\end{array}$ & $\begin{array}{l}\text { Changes the boundary conditions in } \\
\text { Suzen and Huang's model; best suited } \\
\text { for optimization, particularly for } \\
\text { turbine airfoil designs. }\end{array}$ \\
\hline Bouchmal $^{31}$ & $\begin{array}{l}\text { Suzen and Huang's model has been improved; } \\
\text { includes a relationship that incorporates changes in } \\
\text { the voltage to the Debye length as well as changes in } \\
\text { the standard deviation of the Gaussian charge } \\
\text { distribution boundary condition. }\end{array}$ & $\begin{array}{l}\text { Provides relations for the prediction } \\
\text { of characterizing parameters of Suzen } \\
\text { and Huang's model. }\end{array}$ \\
\hline
\end{tabular}

Table 1. Summary of previous models' features and contributions 
wave propagates, attention is usually focused on how the electric field impacts electron motion in the presence of the nucleus, and hence the basic dipole moment of this system. Based on this behavior, models of the medium's electric susceptibility and, as a result, permittivity have been developed. One of the most popular material models is the Lorentz model, which represents the temporal reaction of a component of a medium's polarization field to the same electric field component. According to the Lorentz model, a medium excited by an applied electromagnetic wave is defined by the created polarization field and the region's permittivity. The plasma permittivity, or more accurately, the electromagnetic frequency, plasma frequency, and frequency of electron-neutral collisions, governs wave propagation, evanescence, or attenuation. When it comes to DBD plasma actuators, the characterizing permittivity of the plasmonic region becomes attenuating, introducing the region as a dispersive medium. In this model, this dispersed energy is expressed in terms of a volume body force. It is then incorporated into the Navier Stokes equations to mimic the energy transfer to the fluid flow.

\section{Body Force Formulation}

The electrohydrodynamic (EHD) force is defined as,

$$
\vec{f}_{b}=\rho_{c}(\vec{E}+\vec{V} \times \vec{B})
$$

where, $\vec{f}_{b}$, is the body force per unit volume, $\rho_{c}$, is net the charge density, $\vec{E}$, is the electric field, $\vec{V}$, is the velocity vector, and $\vec{B}$, is the magnetic field.

In general, this system can be explained using four Maxwell equations:

$$
\begin{aligned}
& \operatorname{curl} \vec{H}=\vec{j}+\frac{\overrightarrow{\partial D}}{\partial t} \\
& \operatorname{curl} \vec{E}=-\frac{\overrightarrow{\partial B}}{\partial t} \\
& \operatorname{div} \vec{D}=\rho_{c} \\
& \operatorname{div} \vec{B}=0
\end{aligned}
$$

The influence of the plasma actuator on the flow is investigated numerically, first by analyzing the energy transfer process. The influence of this energy addition is then attempted to be imitated as accurately as possible. Since the electric field generation and ion rearrangement are much faster than the flow response, ${ }^{33}$, the energy generation part can be considered as a quasi-stable process. Therefore, the ions' arrangement will be considered constant, and the current will be zero. Also, all time-derivatives in the above equations become zero, and the only remaining equation will be,

$$
\operatorname{div} \vec{D}=\rho_{c}
$$

The electric induction vector, $\vec{D}$, is connected to the electric field through the dielectric coefficient. Electric induction represents the force induced to dielectric by the electric field,

$$
\vec{D}=\varepsilon \vec{E}
$$

Moreover, the above equation yield that the gradient of a scalar potential can be used to calculate the electric field,

$$
\vec{E}=-\vec{\nabla} \varphi
$$

Gauss's law yields,

$$
\nabla(\varepsilon \nabla \varphi)=-\rho_{c}
$$

where $\varepsilon$ is the permittivity. The permittivity can be expressed as,

$$
\varepsilon=\varepsilon_{r} \varepsilon_{0}
$$


where $\varepsilon_{r}$, is the relative permittivity of the medium, and $\varepsilon_{0}$, is the permittivity of the free space. The Lorentz model then defines the temporal response of a component of the medium's polarization field to the corresponding component of the electric field, assuming that the charges are allowed to travel in the same direction as the electric field as,

$$
\frac{d^{2}}{d t^{2}} P_{i}+\Gamma_{L} \frac{d}{d t} P_{i}+\omega_{0}^{2} P_{i}=\varepsilon_{0} \chi_{L} E_{i}
$$

The expression gives the frequency response, assuming the standard $\exp (+j \omega t)$ time dependency, as,

$$
P_{i}(\omega)=\frac{\varepsilon_{0} \chi_{L} E_{i}}{-\omega^{2}+j \Gamma_{L} \omega+\omega_{0}^{2}}
$$

The response is resonant at the natural frequency with negligible losses $f_{0}$. The electric susceptibility is related to polarization and electric fields as,

$$
\chi_{e, \text { Lorentz }}(\omega)=\frac{P_{i}(\omega)}{\varepsilon_{0} E_{i}(\omega)}=\frac{\chi_{L}}{-\omega^{2}+j \Gamma_{L} \omega+\omega_{0}^{2}}
$$

The permittivity then comes immediately as,

$$
\varepsilon_{\text {Lorentz }}(\omega)=\varepsilon_{0}\left[1+\chi_{e, \text { Lorentz }}\right]
$$

The restoring force is insignificant in a lightly ionized region. As a result, the Drude model is obtained, represented as,

$$
\chi_{e, \text { Drude }}(\omega)=\frac{\chi_{D}}{-\omega^{2}+j \Gamma_{L} \omega}
$$

where the plasma frequency, $\omega_{p}=\sqrt{\frac{e^{2} n_{e}}{\varepsilon_{0} m_{e}}}$, generally represents the coupling coefficient, $\chi_{D}=\omega_{p}{ }^{2}$. As previously noted, plasma frequency is determined by electron density, $n_{e}$, and the charge of an electron, e, the free space permittivity, $\varepsilon_{0}$, and the electron mass, $m_{e}$. The electron density changes within the range of $10^{17}-10^{20} \mathrm{~m}^{-3}$ based on the gas flow pressure. Hence, considering the assumptions and simplifications above, the resultant volume body force is expressed as,

$$
\vec{f}_{b}=\left(\nabla\left(\varepsilon_{\text {Lorentz }} \nabla \varphi\right)\right) \times \vec{E}
$$

In the Navier Stokes equation, these are expressed as volume body force components. Details of plasma dynamics that would have added complexity and computing cost in prior models have been implemented using this approach to develop a criterion for the plasma to generate fluid flow.

\section{The numerical simulation method and the non-dimensionalized form}

Since the time dependency is caused solely by the boundary condition for the applied voltage at the exposed electrode, by applying a constant boundary condition, the related equations and voltage boundary conditions are rendered time-independent and solved. The following are the normalized parameters for 2-D coordinates:

$$
\begin{aligned}
& \phi=\frac{\varphi}{f(t)} \\
& \vec{E}=\nabla \phi
\end{aligned}
$$

Where $f(t)$ is a function that represents the voltage's waveform. Furthermore, for fluid flow modeling, the 2-D incompressible Reynolds Averaged Navier-Stokes (RANS) equations are utilized. It is anticipated that the bulk of the energy provided by the plasma actuator is mostly used to accelerate the fluid particles; thus, the amount contributing to fluid warming is deemed unimportant, and the flow field energy equation is neglected ${ }^{40}$. The following are the fundamental equations of momentum and mass conservation that were used for fluid flow simulation:

$$
\nabla \cdot \vec{u}=0
$$

$$
(\vec{u} . \nabla) \vec{u}=-\frac{1}{\rho} \nabla P+v \nabla^{2} \vec{u}+\vec{f}_{b}
$$

where $\rho, \vec{u}, v$, and $P$ are the density, velocity, kinematic viscosity, and static pressure, respectively, and $\vec{f}_{b}$ is the body force per unit volume in $N /\left(\mathrm{m}^{3}\right)$. In Eq.(16), the volume body force vector components created by plasmonic actuation are added to the momentum equation's right-hand side (Eq.20). 


\section{Model Implementation}

In order to accurately imitate the influence of the plasma actuator on flow dynamics, the domain addressed to the plasma must have two characteristics: first, it must be compatible with plasma formation physics, and second, it must be self-scalable. When compared to a simple parallel plane structure, the existence of a dielectric between the electrodes and the asymmetry results in a qualitatively different pattern of electric field lines in an asymmetric actuator configuration. The electric field is most intense near the inner margins of the two electrodes. As a result, plasma generation is more likely in areas with stronger electric fields. In this layout, the anode surface is the dielectric rather than the electrode, resulting in a pseudo anode. In this regard, the anode region directly adjacent to the pseudo anode is an electron-rich zone. Since the dielectric, in contrast to the cathode, prohibits charge mobility on its surface, we have a concentration of charges on the cathode and a comparatively sparse distribution of electrons on the pseudo anode ${ }^{23}$. The Debye length, which is the general characteristic length scale for plasma discharges, is another length that contributes to the plasmonic area. Based on the foregoing, the domain of the plasmonic area is defined as a rectangle with a height equal to the Debye length and a width beginning at a portion of the exposed electrode equal to the Debye length and ending at the trailing edge of the embedded electrode. Figure 2 illustrates the described plasmonic region. This region provides the self-scalability for the model. However, the nature of the plasma is considered to provide constant permittivity for the entire plasmonic domain for the sake of simplicity. The more accurate distribution of the permittivity, as well as its dependency on the applying voltage and the exciting frequency, is going to be undertaken in future research. The Debye length $\lambda_{D}$ is given from the following empirical relation ${ }^{29,30}$ :

$$
\lambda_{D}(m)=0.2\left(0.5611 \times \tan ^{-1}\left(-170.3 \times f(k H z)^{-5.124}\right)+1.768\right) \times\left(0.3 \times 10^{-3} \times V_{a p p}(k V)-7.42 \times 10^{-4}\right)
$$

It is clear that based on the previous equation, the Debye length changes due to the change in the applied voltage and the exciting frequency. Since the area considered as the plasmonic region is defined based on the Debye length, this area will change as a consequence of changes in the voltage and frequency.

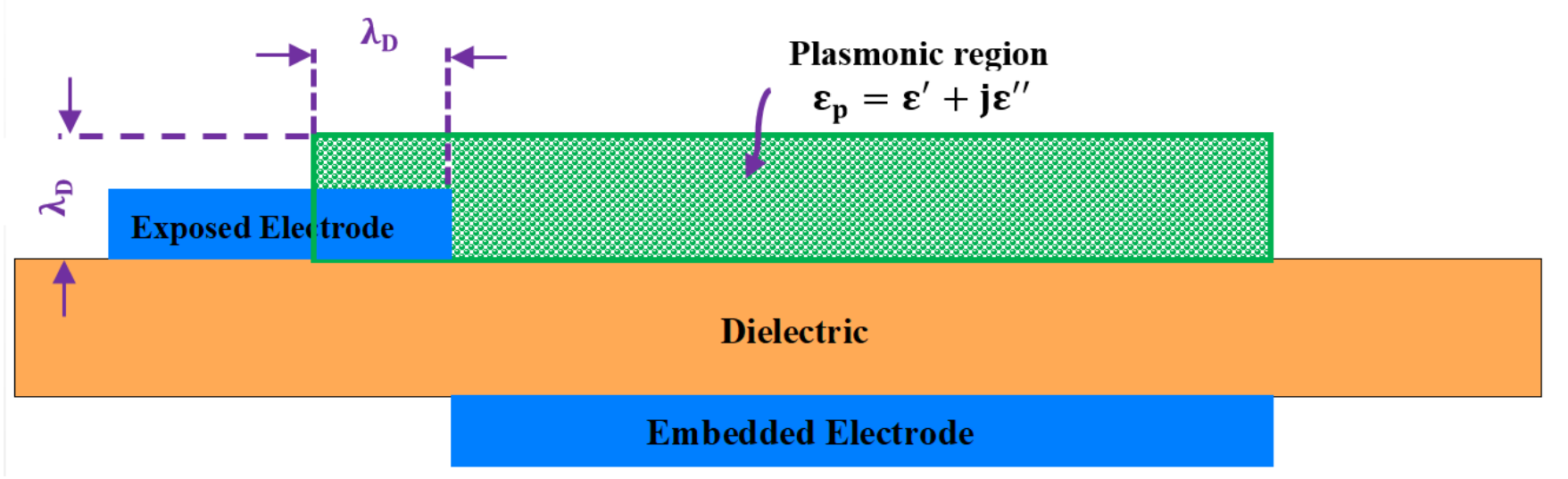

Figure 2. The schematic of a DBD plasma actuator to illustrate the defined plasmonic region by the presented model

\section{Model Limitations}

Intensive work has been done to model the fluid flow impacted by the plasma actuators as accurately as possible while keeping the computational cost near the optimum. These models give basic algorithms for simulating plasma actuators. However, they disregard the intricacies of plasma creation, modeling the integral effects of the plasma jet on the flow to avoid the complexity and high computational expense of a proper investigation of the physical phenomena. Furthermore, these models rely on experimental data to change their characteristic properties. Various formulations have been offered in the literature $20,23-26,30,31,33,34$. These formulations have become optimized and applicable yet add to the complexity of the model, and still avoid the implementation of the plasma physics. Details of plasma dynamics have been implemented using the provided approach to establish a criterion for the plasma to generate fluid flow while keeping the model simple and low in computational cost compared to fundamental principle-based models. Nonetheless, the proposed model is currently being refined to provide complete controllability over the actuating body force components. Furthermore, the model is restricted to the frequency $(1 \mathrm{kHz}-14 \mathrm{kHz})$, and the applied voltage $(3 \mathrm{kVpp}-20 \mathrm{kVpp})$ ranges. While experience has shown that the ranges are adequate for most engineering applications, more precise reasoning will be pursued in future studies. 


\section{Boundary Condition}

Figure 3 illustrates the boundary conditions for the solution of the Poisson equation. The electric potential equation and the Gauss Law's equation (Eq.9) is solved in the outer domains and the outer boundaries. As mentioned earlier, the equations and the voltage boundary conditions are modified to be time-independent; therefore, $\phi=0$ is set on the embedded electrode, and $\phi=\phi_{\max } / \sqrt{2}=\phi_{r m s}$, on the exposed electrode. $\phi_{\max }$ refers to the amplitude of the applied AC voltage.

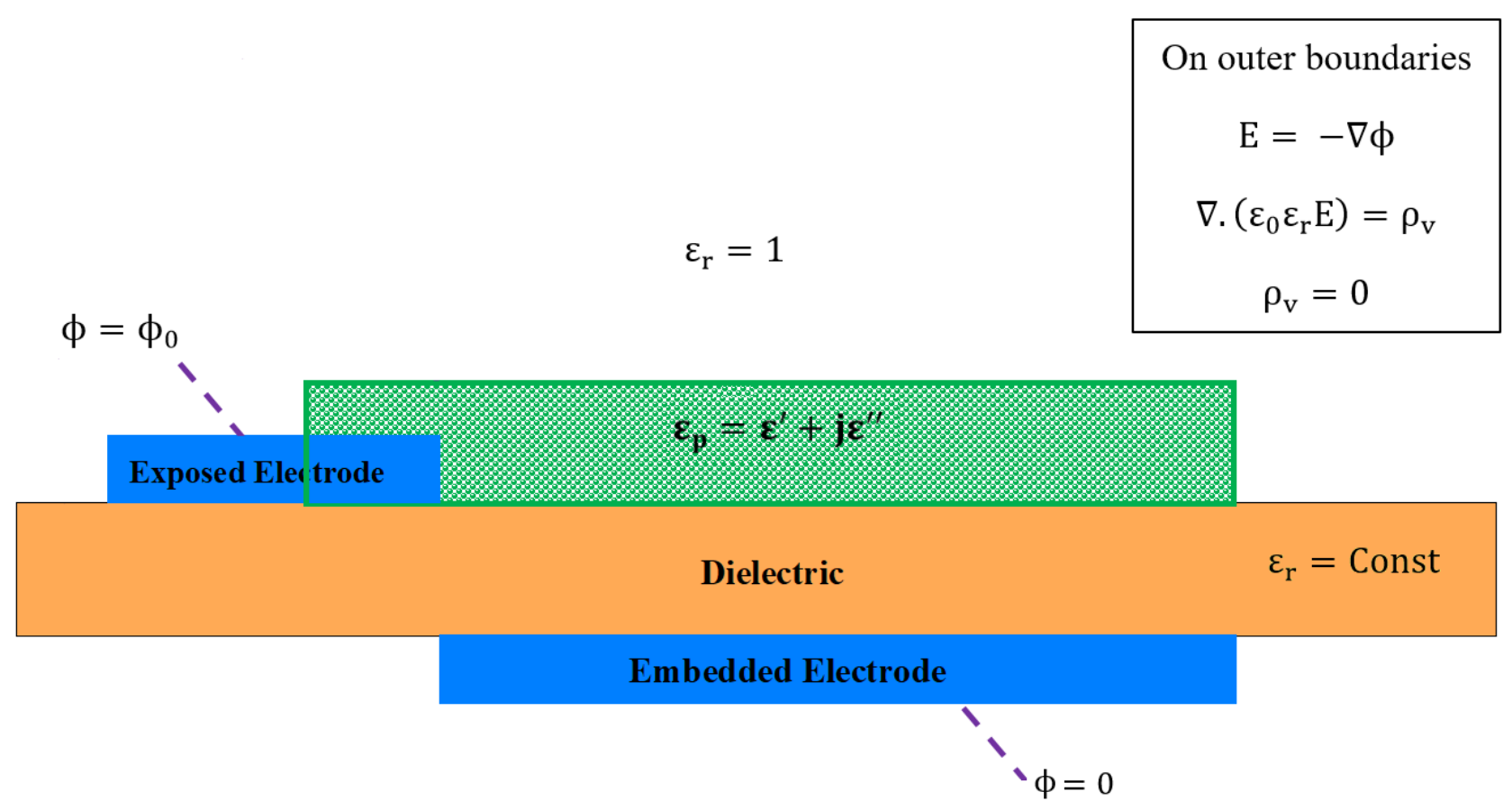

Figure 3. A DBD actuator schematic including the boundary conditions for electrostatic simulation

\section{Results and Discussion}

An experiment has been conducted to fully explore the performance of the presented model on predicting the effects of a plasma actuator on fluid flow. Furthermore, to analyze the applicability and adjustability of the model, the experimental case from Kotsonis et al. ${ }^{41}$ has been selected for the study. A final comparison was made based on an experiment by Palmeiro et $\mathrm{al}^{42}$ regarding nominated numerical modeling cases with different actuator configurations and applied voltages and excitation frequencies. These studies provide a comprehensive understanding of the applicability of the presented model based on the experimental data and a thorough comparison concerning other numerical approaches. The reports presented in the following are based on simulations to ensure the validity of the discussion. For simulation purposes, the reference geometry of the DBD configuration varies depending on the chosen situations. These geometries are considered 2-D shapes for the simulation, which is a reasonable assumption given the considerable length to thickness ratio of all DBD combinations. The meshing was with triangular pieces, and the mesh setting was initially set to "extremely fine." The grid spacing was limited to no more than the Debye length. Furthermore, an adaptive mesh refinement was used to achieve grid independence of the acquired results while also minimizing the numerical cost for the simulation of each case to provide perfection with the meshing based on the multiphysics nature of each problem. Table 2 contains the test conditions and experimental data for all of the conducted experiments as well as the study cases. In all cases, the working fluid is air at standard condition $\left(v=1.75 e 5 \mathrm{~m}^{2} \mathrm{~s}^{-2}\right.$ and $\left.\rho=1.18 \mathrm{kgm}^{-3}\right)$.

Results from conducted experiments. The obtained results from the conducted experiments are presented in this section. Details regarding the experimental setup and measurement systems are discussed in the section titled "Method." Figure 4 provides the configuration used for the experiment. The velocity profiles are the metric used to assess the efficacy of the proposed model in predicting the strength of the simulated induced jet and its interaction with the neighboring fluid in comparison to the experimental data. The Applied voltages and frequencies for the experiments and the corresponding simulations are 6,7.2 kVpp and $6,8 \mathrm{kHz}$, respectively. As mentioned in Table 2 the length of the exposed and embedded electrodes are $10 \mathrm{~mm}$ and $30 \mathrm{~mm}$, 


\begin{tabular}{|c|c|c|c|c|c|}
\hline \multirow{2}{*}{ Specifications } & \multirow{2}{*}{ Experiment } & \multirow{2}{*}{ Kotsonis et al. ${ }^{41}$} & \multicolumn{3}{|c|}{ Palmeiro et al. } \\
\cline { 4 - 6 } & & & Case A & Case B & Case C \\
\hline Exposed Electrode Length (lex) & $10 \mathrm{~mm}$ & $10 \mathrm{~mm}$ & $6.35 \mathrm{~mm}$ & $12.7 \mathrm{~mm}$ & $5 \mathrm{~mm}$ \\
\hline Embedded Electrode Length (lem) & $30 \mathrm{~mm}$ & $10 \mathrm{~mm}$ & $6.35 \mathrm{~mm}$ & $12.7 \mathrm{~mm}$ & $5 \mathrm{~mm}$ \\
\hline Electrode Gap (lg) & $0 \mathrm{~mm}$ & $0 \mathrm{~mm}$ & $1 \mathrm{~mm}$ & $1 \mathrm{~mm}$ & $0 \mathrm{~mm}$ \\
\hline Electrode Thickness (te) & $0.05 \mathrm{~mm}$ & $0.06 \mathrm{~mm}$ & $0.074 \mathrm{~mm}$ & $0.074 \mathrm{~mm}$ & $0.074 \mathrm{~mm}$ \\
\hline Dielectric Thickness (td) & $0.6 \mathrm{~mm}$ & $0.11 \mathrm{~mm}$ & $0.19 \mathrm{~mm}$ & $0.57 \mathrm{~mm}$ & $0.18 \mathrm{~mm}$ \\
\hline Dielectric Cosntant & 2.7 & 2.7 & 2.9 & 2.9 & 2.9 \\
\hline
\end{tabular}

Table 2. The nominal experimental conditions and parameters as well as specifications and conditions for the study cases

respectively. The thickness of the electrodes is $0.05 \mathrm{~mm}$, and the dielectric is made up of polyimide Kapton tape with a total thickness (including adhesive layer) of $0.6 \mathrm{~mm}$ and relative permittivity of 2.7 .

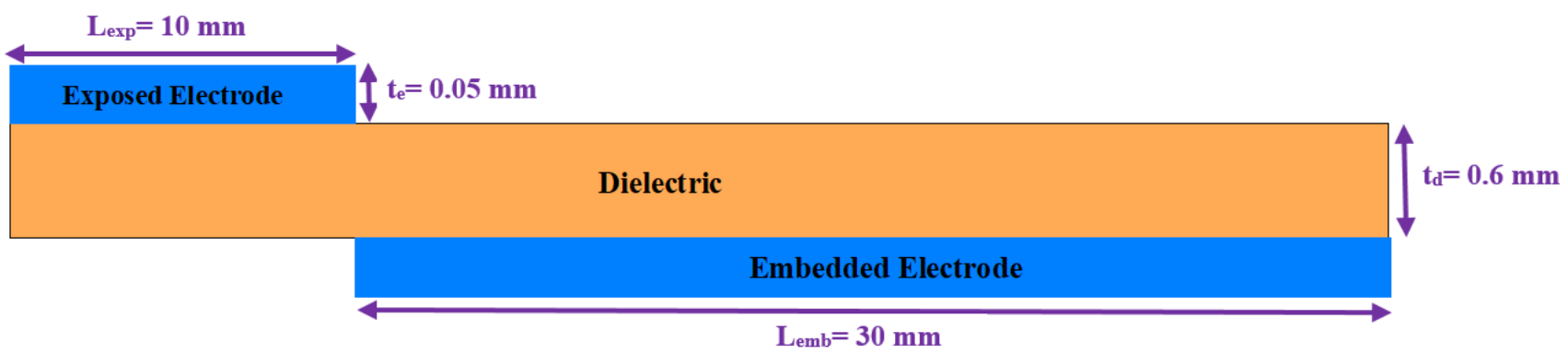

Figure 4. The actuator arrangement utilized in the studies is depicted schematically

For this scenario, the computing domain is a rectangle $104 \mathrm{~cm}$ long and $50 \mathrm{~cm}$ high. The bottom boundary condition is set to no-slip, and the upper boundary is subject to the symmetry criterion. The velocity at the left inlet boundary is set to be zero, and the pressure at the right outlet boundary is set to be zero, as well. The actuator is adjusted at around $30 \%$ near the inflow boundary.

Figure 5, represent the induced velocity profiles obtained using the current model, compared to the experimental data at a station $5 \mathrm{~mm}$ downstream of the exposed electrode's leading edge for the applied voltages of $6 \mathrm{kVpp}$ and the excitation frequency of $6,8 \mathrm{kHz}$, respectively. Based on the flow characteristics, the Debye length (21) is calculated to be $0.114 \mathrm{~mm}$ and $0.118 \mathrm{~mm}$, respectively. It is observed that although the presented numerical model underestimates the scaled velocity, it is capable of capturing the general trend of the velocity profile. Moreover, the model estimates the height where the maximum velocity occurs, characterizing the jet-induced boundary layer thickness with acceptable accuracy.

Figure 6 provides the electric potential field around the actuator. The maximum voltage difference is observed between the edges of the embedded and exposed electrodes, resulting in the maximum electric field magnitude. Figure 7 provides the flow velocity field around the vicinity of the actuator, indicating that the majority of momentum increases occur in the x-direction. A weak suction effect is also found upstream of the inner edge of the electrodes, indicating the presence of a potentially large pressure differential near the actuator.

Figure 8 provides the induced velocity profiles for the applied voltages of $7.2 \mathrm{kVpp}$ and the excitation frequency of 6 , $8 \mathrm{kHz}$, respectively, comparing the current model to the experimental results at a station $5 \mathrm{~mm}$ downstream of the exposed electrode's leading edge. Based on the flow characteristics, the Debye length (21) is calculated to be $0.0228 \mathrm{~mm}$ and $0.0229 \mathrm{~mm}$, respectively. The presented results show that the numerical model is able to accurately simulate the general trend of the velocity profile as well as predict the height where the maximum velocity occurs.

Same as the previous cases, Figure 9 provides the electric potential field around the actuator. It is observed that the maximum voltage difference occurs between the edge of the embedded and exposed electrodes resulting in the maximum electric field magnitude. Figure 10 represents the flow velocity field around the vicinity of the actuator, showing that the momentum increases mainly in the x-direction, and a potentially strong pressure gradient due to a weak suction effect exists upstream the inner edge of the electrodes. 


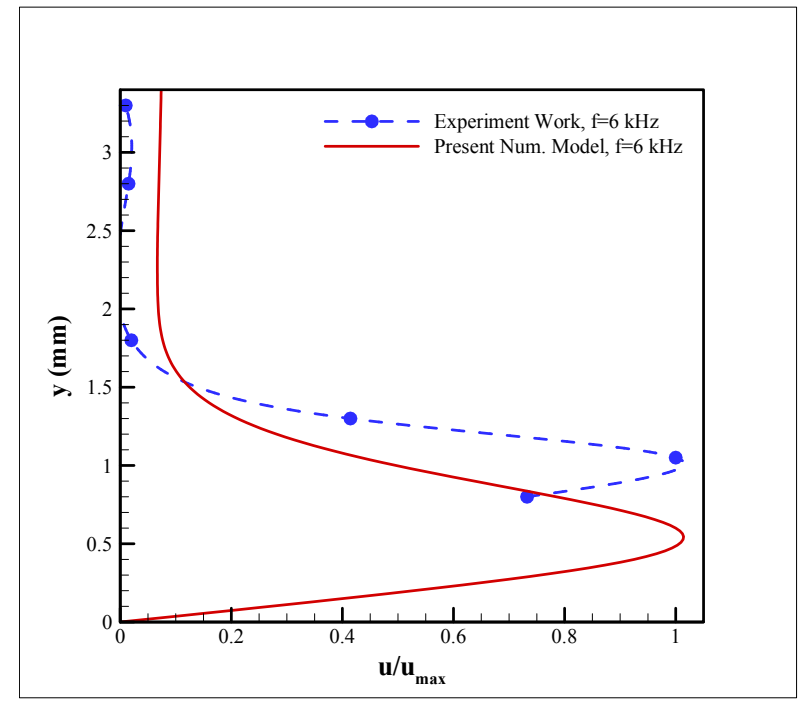

(a)

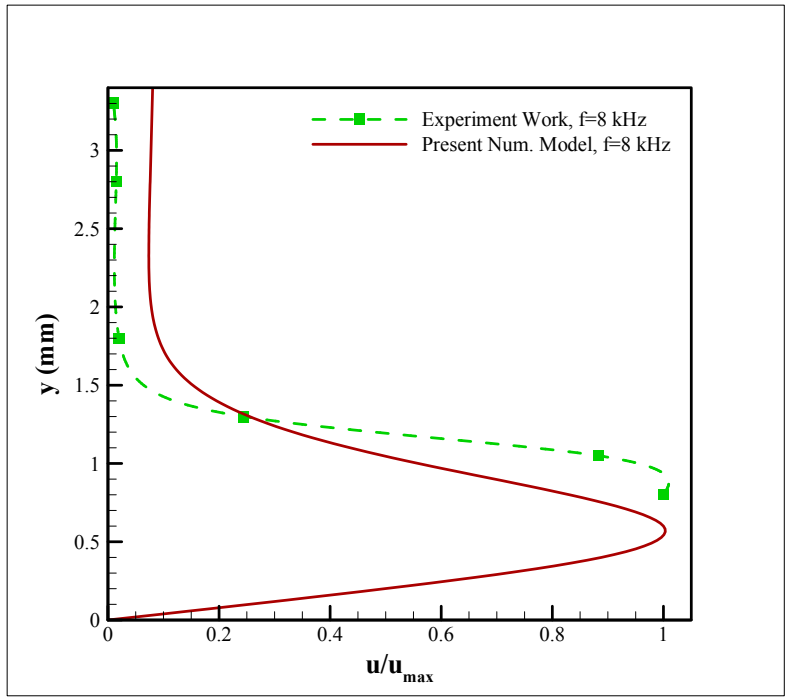

(b)

Figure 5. Comparison of velocity profiles from the experiments and the presented numerical scheme for (a) $\mathrm{V}=6 \mathrm{kVpp}$, $\mathrm{f}=6 \mathrm{kHz}$, and $(\mathrm{b}) \mathrm{V}=6 \mathrm{kVpp}, \mathrm{f}=8 \mathrm{kHz}$.

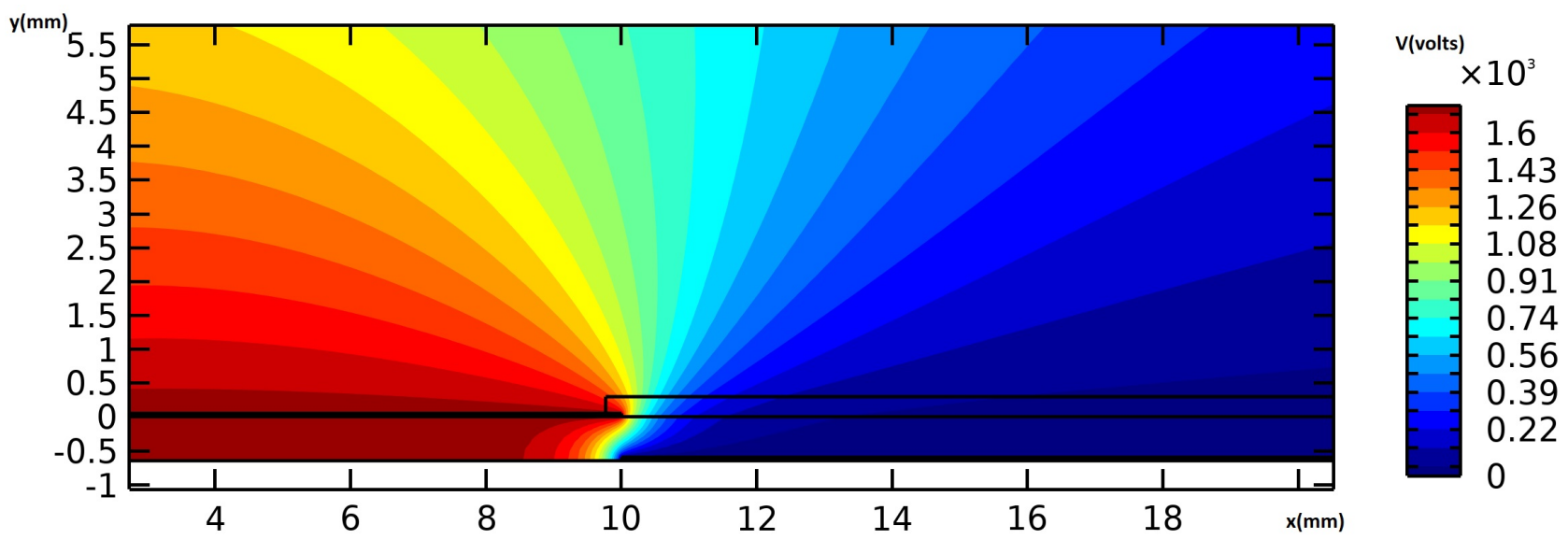

Figure 6. The electric potential field around the actuator in volts, for the actuation case of $\mathrm{V}=6 \mathrm{kVpp}$ and $\mathrm{f}=8 \mathrm{kHz}$

The maximum velocities obtained using the presented numerical model and the corresponding results from the experiments are tabulated in Table 3. The results reveal that the model predicts the maximum velocity, as an indication of the total body force, with acceptable accuracy.

\begin{tabular}{|c|c|c|c|c|}
\hline $\mathrm{V} \mathrm{kV}$ & $6 \mathrm{kHz}$ - Current Model & $6 \mathrm{kHz}$ - Experiment & $8 \mathrm{kHz}$ - Current Model & $8 \mathrm{kHz}$ - Experiment \\
\hline 6 & 0.73 & 0.74 & 0.71 & 0.73 \\
\hline 7.2 & 1.16 & 1.83 & 1.03 & 1.10 \\
\hline
\end{tabular}

Table 3. The comparison of the calculated integrated horizontal body force component for different input voltages and the experimental results

The Experiment of Kotsonis et al.. The experiment of Kotsonis et al. ${ }^{41}$ on the body force field of DBD actuators is selected to serve as a benchmark, providing certainty with the model. Kotsonis et al. provide body force fields derived from PIV observations for various input voltages. The details of the plasma actuator configuration used in the work of Kotsonis et al. are presented in Table2. The plasma actuator configuration used by Kotsonis et al. consists of electrodes with a width of $10 \mathrm{~mm}$ and 


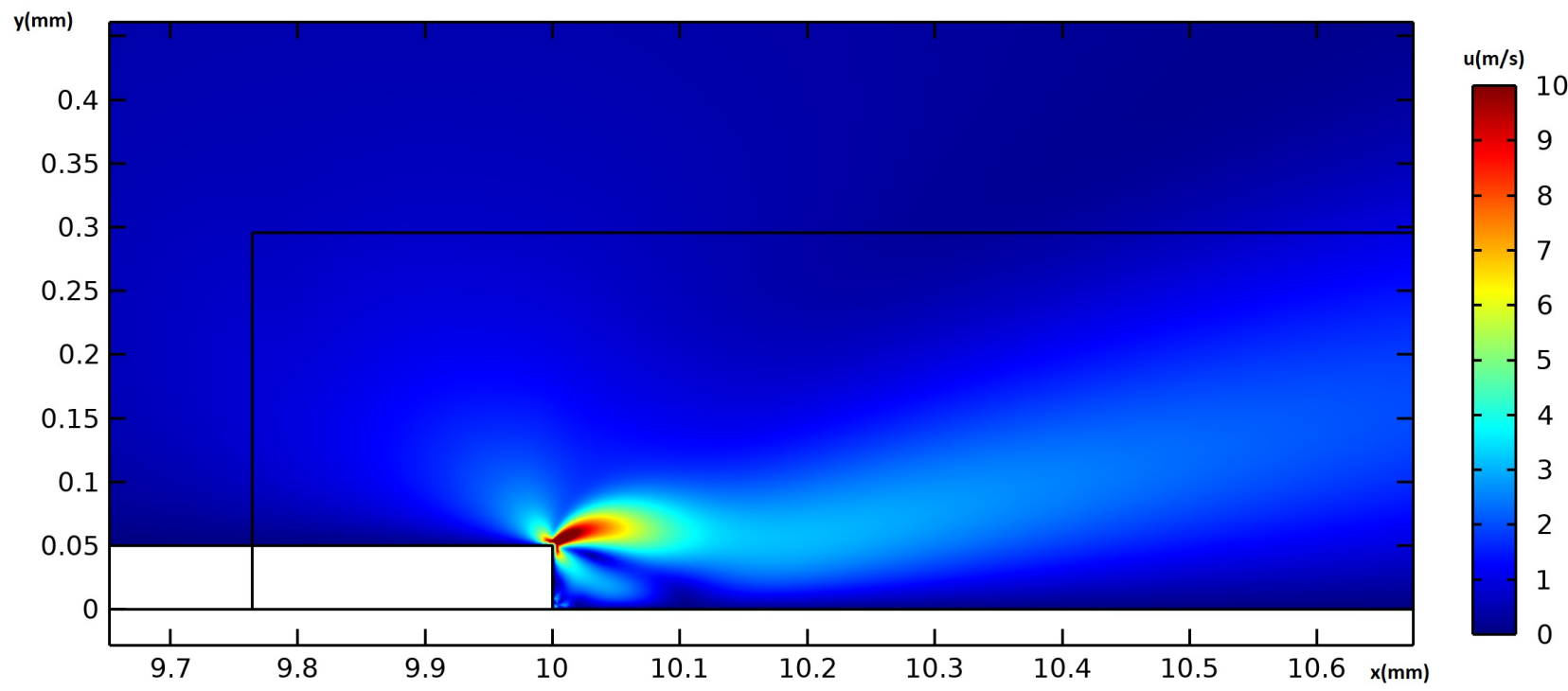

Figure 7. The flow velocity field around the actuator in $m s^{-1}$, for the actuation case of $\mathrm{V}=6 \mathrm{kVpp}$ and $\mathrm{f}=8 \mathrm{kHz}$

$0.06 \mathrm{~mm}$ thickness. The electrodes are separated by a horizontal gap of zero. Furthermore, two dielectric layers of polyimide Kapton tape with a total thickness (including the adhesive layer) of $0.11 \mathrm{~mm}$ separate the electrodes. The peak-to-peak voltage on the electrodes was adjusted from $8 \mathrm{kVpp}$ to $16 \mathrm{kVpp}$ in $2 \mathrm{kVpp}$ steps. For each input peak-to-peak voltage, the body force field is monitored.

The computational domain and the boundary conditions are set to be the same as in the previous section. Based on the flow characteristics provided by Kotsonis et al., the Debye length is considered to be $2 \mathrm{~mm}$. In this regard, the area considered as the plasmonic region would be a rectangle with the height of the Debye length and a width starting from a portion of the exposed electrode that is equal to the Debye length and ends at the trailing edge of the embedded electrode.

Figures 11 provides the spatial distribution of the body force components based on the presented numerical model. It is observed that the maximum horizontal body force is produced at the edge of two electrodes where, as predicted, is the region with the highest electric field magnitude, therefore, with the most probability for ionization.

Table 4 compares the computed integrated horizontal body force component to the experimental results of Kotsonis et al. for various input voltages, respectively. A comparison is made based on the integrated body forces on the area surrounding the plasmonic region. The Kotsonis et al. experiment area was a rectangle with the height of the Debye length's order and a width starting from $10 \%$ of the exposed electrode and ends at $70 \%$ from the trailing edge of the embedded electrode. One may interpret the results by saying that the presented model is capable of accurately predicting the integral effect of the body force production. The resultant body forces are accurately predicted with a maximum deviation of $7.69 \%$ from the experimental results.

\begin{tabular}{|c|c|c|c|}
\hline $\operatorname{Vmax}(\mathrm{kV})$ & $\mathrm{fx}(\mathrm{N})$ - experiment & $\mathrm{fx}(\mathrm{N})$ - Numerical & Error (\%) \\
\hline 4 & 0.00006 & 0.000058 & 3.33 \\
\hline 5 & 0.0013 & 0.0012 & 7.69 \\
\hline 6 & 0.0032 & 0.0031 & 3.13 \\
\hline 7 & 0.0051 & 0.005 & 1.96 \\
\hline 8 & 0.0093 & 0.0089 & 4.30 \\
\hline
\end{tabular}

Table 4. The comparison of the calculated integrated horizontal body force component for different input voltages and the experimental results

The Work of Palmeiro et al. The final case to examine the applicability of the presented modeling strategy has been selected to be the experimental work of Palmeiro et $\mathrm{al}^{42}$. Furthermore, we compare the current model to various numerical models using the numerical works offered by Palmeiro et al. Following his studies, three test scenarios are explored. The details of all the cases are given in Table 2. For each test case, five sets of results are presented: A) the experiment ${ }^{42}$; B) the lumped-circuit 


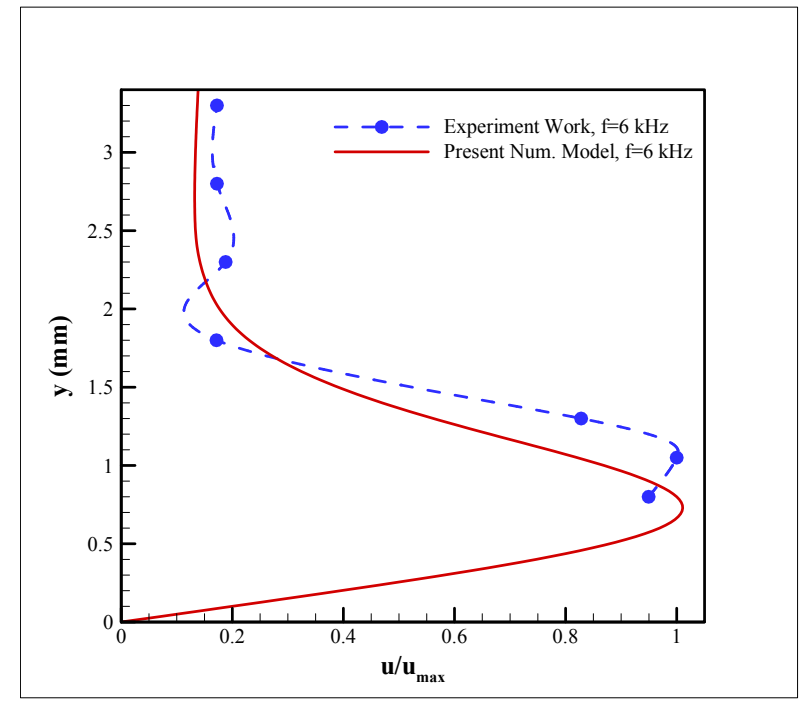

(a)

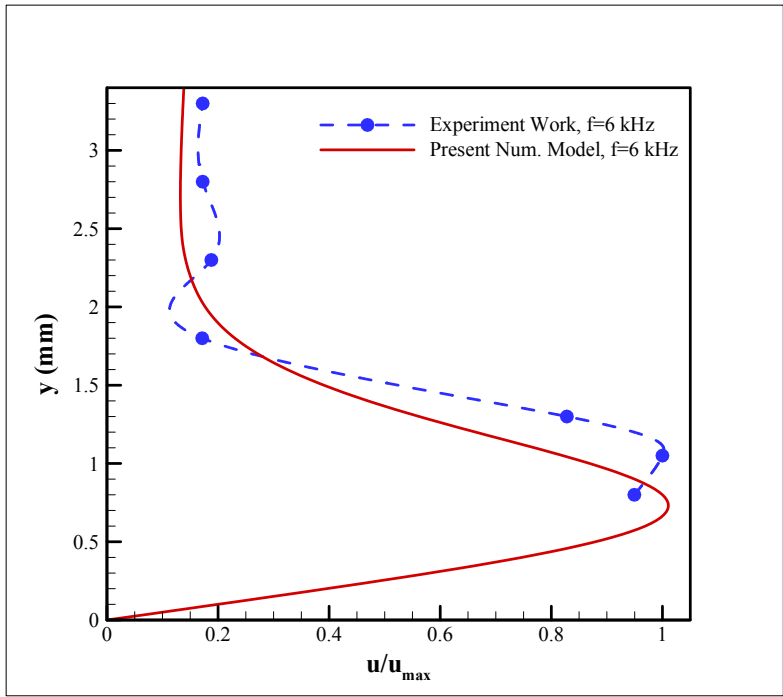

(b)

Figure 8. Comparison of velocity profiles from the experiments and the presented numerical scheme for (a) V=7.2kVpp, $\mathrm{f}=6 \mathrm{kHz}$, and (b) $\mathrm{V}=7.2 \mathrm{kVpp}, \mathrm{f}=8 \mathrm{kHz}$.

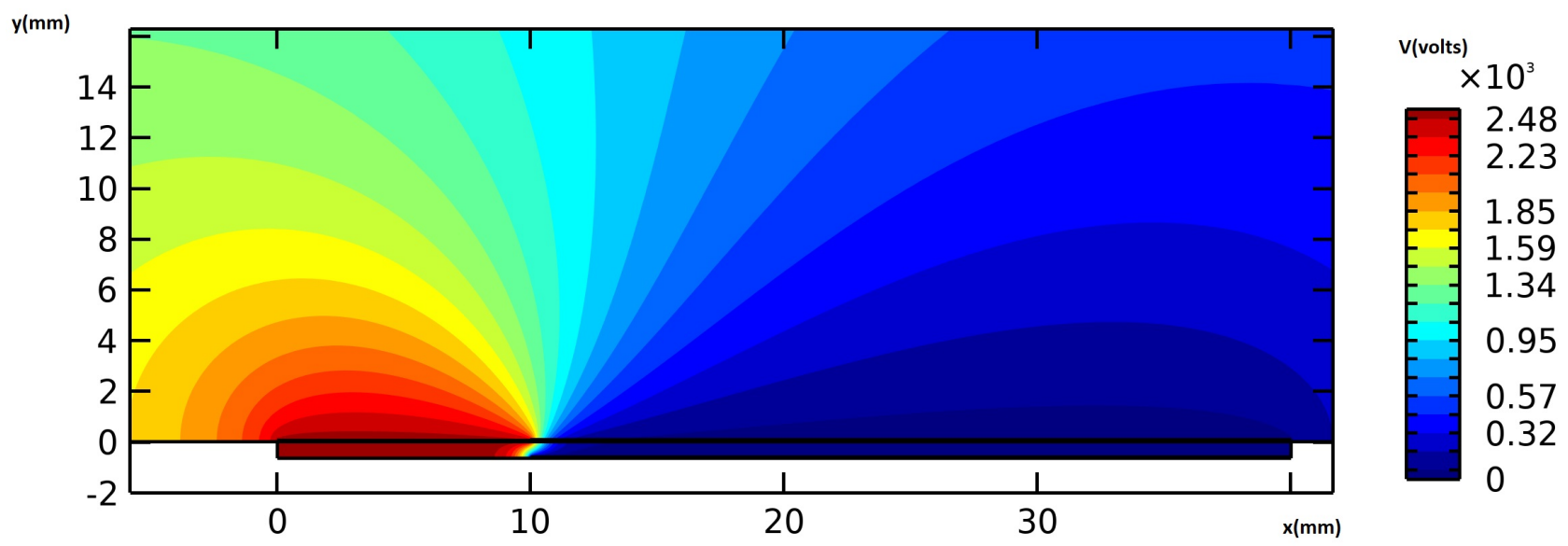

Figure 9. The electric potential field around the actuator in volts, for the actuation case of $\mathrm{V}=7.2 \mathrm{kVpp}$ and $\mathrm{f}=8 \mathrm{kHz}$

$\operatorname{model}^{22}$; C) the hybrid model ${ }^{21}$; D) the simple body force model ${ }^{23}$, and E) the current numerical model. The corresponding maximum velocity normalizes the velocity profiles for every modeling methods. As tabulated in Table 2, the plasma actuator configuration used for cases $\mathrm{A}$ to $\mathrm{C}$ of Palmeiro et al. consists of electrodes with widths of $6.35,12.7$, and $5 \mathrm{~mm}$, respectively, all with a thickness of $0.075 \mathrm{~mm}$. The horizontal gap used between the electrodes is set to be $1 \mathrm{~mm}, 1 \mathrm{~mm}$, and zero, respectively. The electrodes, in all cases, are separated by a dielectric layer of polyimide Kapton tape with a total thickness of $0.19,0.57$, and $0.18 \mathrm{~mm}$, respectively. Moreover, the input peak-to-peak voltage on the electrodes is 12,15 , and $10 \mathrm{kVpp}$, respectively, with excitation frequencies of 3,3 , and $2.75 \mathrm{kHz}$, respectively. The computational domain, as well as the boundary conditions, are set to be the same as in previous sections. Based on the flow characteristics, the Debye length (21) for cases A to C is calculated to be $0.46,0.74$, and $0.28 \mathrm{~mm}$, respectively. In this regard, the area considered as the plasmonic region would be a rectangle with the height of the Debye length and a width starting from a portion of the exposed electrode equal to the Debye length and ends at the trailing edge of the embedded electrode. Figures 12 to 14 provide the induced velocity profiles obtained based on the experimental and the numerical results from Palmeiro et al. compared to the results from the presented numerical model for cases $\mathrm{A}, \mathrm{B}$, and $\mathrm{C}$, respectively.

Based on the results for case A (Figure 12), the presented numerical model performs as well as the Simple Body Force and the Hybrid models in estimating the velocity profile. While the three models over-predict the normalized velocity, the presented 


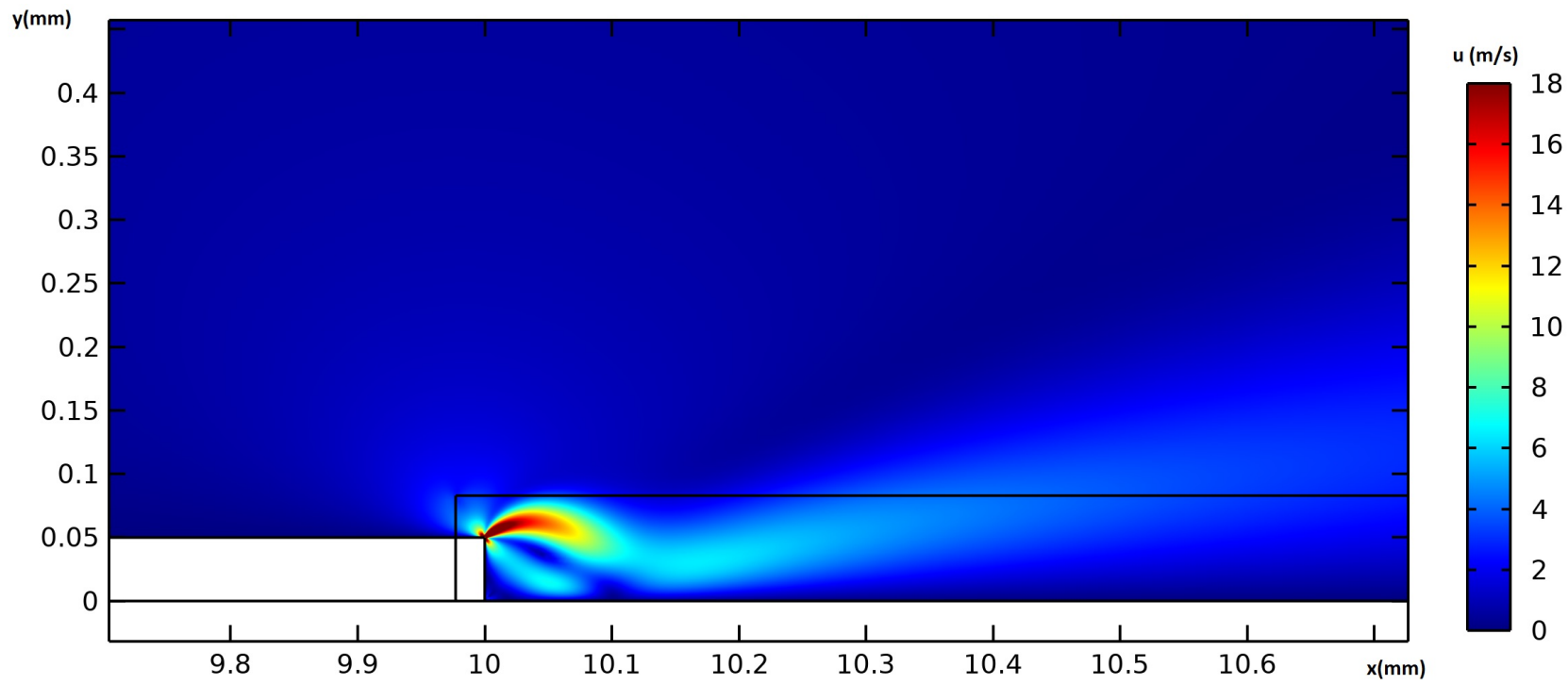

Figure 10. The flow velocity field around the actuator in $m s^{-1}$, for the actuation case of $\mathrm{V}=7.2 \mathrm{kVpp}$ and $\mathrm{f}=8 \mathrm{kHz}$

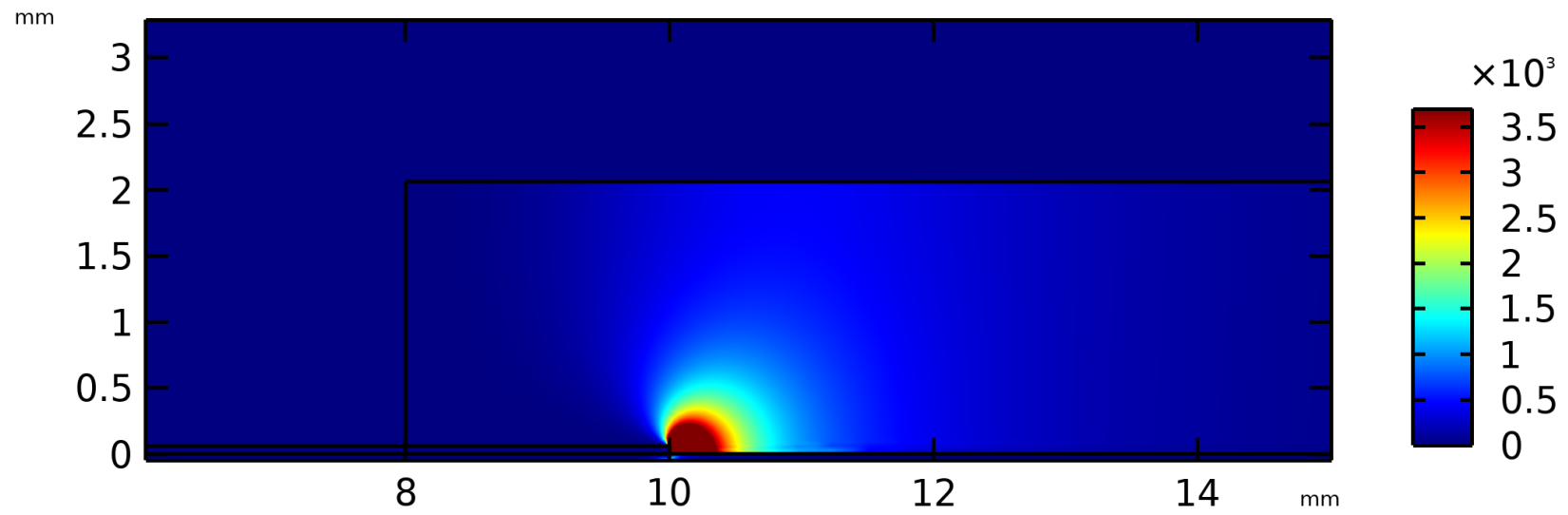

Figure 11. Spatial distribution of the horizontal body force component calculated based on the presented numerical model

model provides the best result. The Lumped Circuit model is the one method that correctly predicts the normalized velocity while it deviates from the experimental results when moving away from the actuator.

Comparing the numerical results to the experimental data in Figure 13 for case B, it is observed that the presented numerical model provides the best prediction of the velocity profile compared to the other numerical schemes. Both the presented numerical model and the Simple Body Force model can accurately predict the jet boundary layer thickness, while the results of the latter deviate from the experimental data as we move away from the actuator. The figure shows that the Hybrid model fails to capture the velocity profile, and the Lumped Circuit model underestimates the normalized velocity although capturing the general trend.

Results from case C (Figure 14) show that the presented numerical model provides the best prediction for the jet boundary layer thickness characterized by the location where the maximum velocity occurs compared to the Simple Body Force and the Lumped Circuit models. However, similar to the Lumped Circuit model, the presented numerical model underestimates the normalized velocity when moving away from the actuator. Unlike the hybrid model, the presented numerical model performs as well as the Simple Body Force and the Lumped Circuit models in capturing the general trend of the velocity profile.

Once the findings from all three examples are reviewed, it is clear that the given modeling method has the most consistent predictive capability for the various test cases compared to the other phenomenological models. 


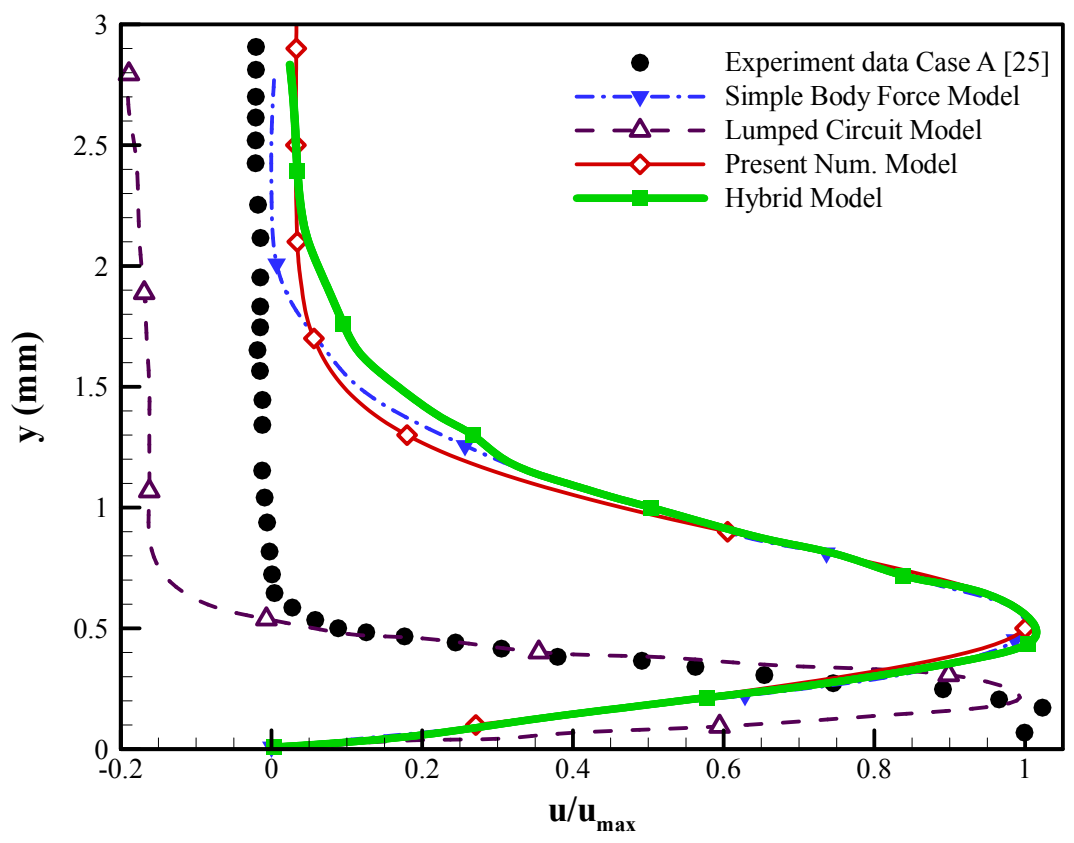

Figure 12. The results from the presented numerical model compared to the experimental and other numerical vertical velocity profiles on actuator geometry of Case A

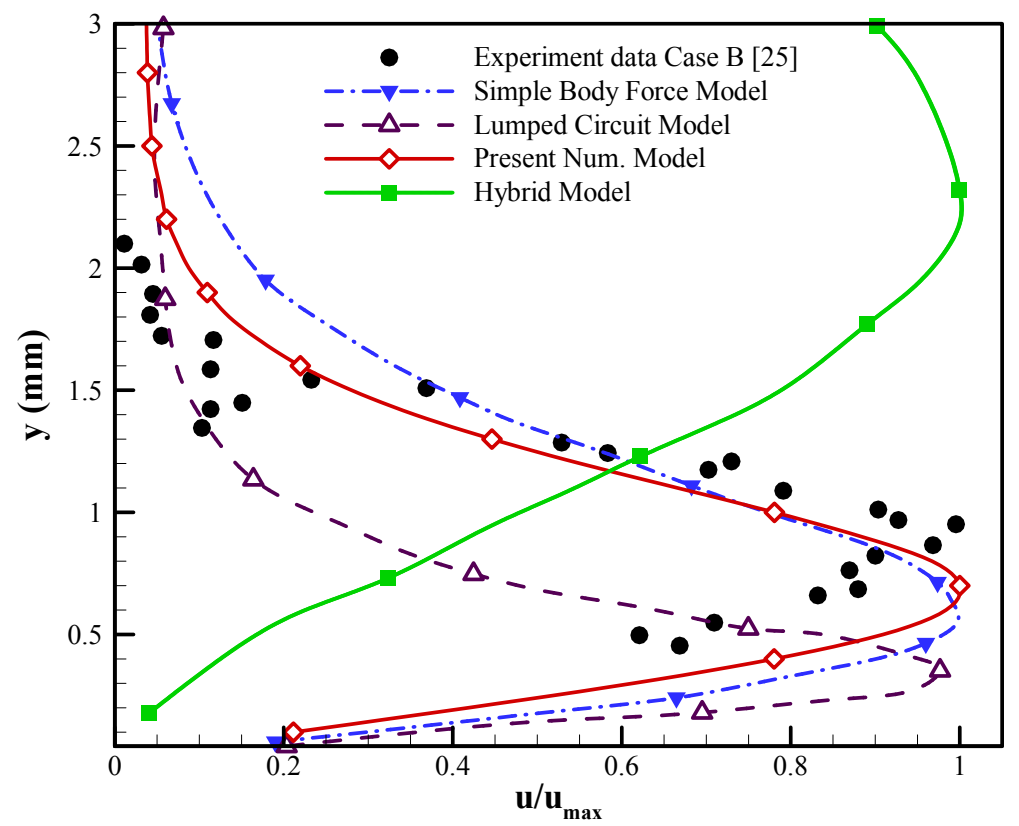

Figure 13. The results from the presented numerical model compared to the experimental and other numerical vertical velocity profiles on actuator geometry of Case B

To compare the potentiality of the presented model to other numerical methods more in detail, Table 5 offers the maximum velocity obtained at $\mathrm{x}=25 \mathrm{~mm}$ from the exposed electrode's leading edge, respectively, for cases A, B, and C. The present model 


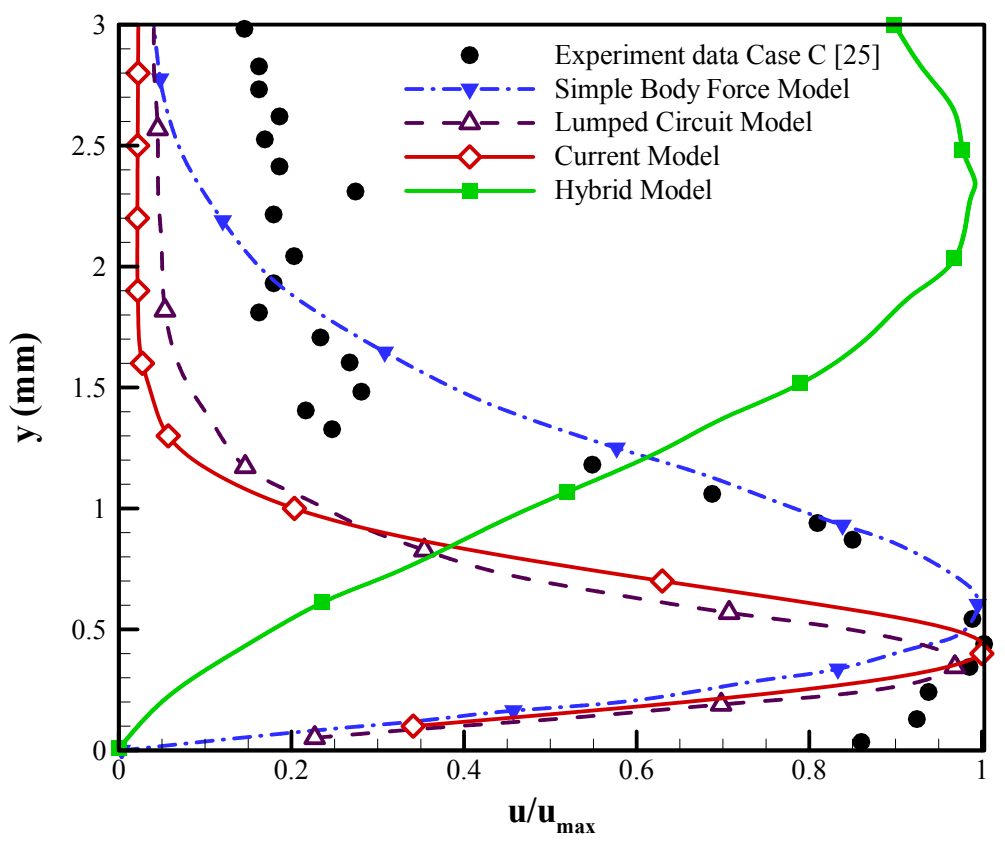

Figure 14. The results from the presented numerical model compared to the experimental and other numerical vertical velocity profiles on actuator geometry of Case $\mathrm{C}$

\begin{tabular}{|c|c|c|c|c|c|}
\hline Case Study & Experiment $^{42}$ & Lumped-Circuit Model $^{22}$ & Hybrid Model $^{21}$ & Simple Body Force Model $^{23}$ & Current Model \\
\hline Case A & 1.9458 & 8.9311 & 1.5339 & 0.7266 & 2.7141 \\
\hline Case B & 1.2772 & 7.3674 & 0.2927 & 0.8383 & 2.3714 \\
\hline Case C & 1.5181 & 3.977 & 1.2382 & 0.3042 & 5.0699 \\
\hline
\end{tabular}

Table 5. The computed maximum velocity achieved at $x=25 \mathrm{~mm}$ from the leading edge of the exposed electrode, compared to numerical technique outcomes for three cases of A, B, and C.

shows the most consistent predictive capability and adequate accuracy for all three cases in comparison to other numerical scenarios.

\section{Conclusion}

In this study, a new modeling approach has been outlined for the numerical investigation of the fluid flow affected by a plasma actuator. In this new approach, we presented a model that simulates the plasmonic region based on the practical material model, the Lorentz model, to characterize the region as a dispersive medium. The dissipated energy added to the flow is calculated in terms of local body force components by solving Poisson's equation for the electric field and implementing the simplified Lorentz model for the polarization field. The area considered as the plasmonic region is defined to be based on the characterizing Debye length, changing as a function of the excitation frequency and the applied voltage. In this regard, the current approach establishes a criterion for the plasma to induce the fluid flow by considering the details of the plasma dynamics. This facilitated us to define the characterizing parameters of the actuation to be self-adjustable based on the physics while keeping the model simple and of low computational cost compared to the fundamental principle-based models. We conducted an experiment to compare the observed influence of plasma actuators on fluid flow with the results predicted by the model to assess the proposed model's validity and performance. The results indicated that the model could capture the general trend of the velocity profile and estimate the jet-induced boundary layer thickness with acceptable accuracy. The model also estimated the significant increase in momentum in the $\mathrm{x}$-direction regarding the actuator and a potentially significant pressure differential near the actuator. Furthermore, the model's universality was validated using diverse experiments and exempted 
numerical models. The integral effects of plasma actuation have been predicted with a maximum error of roughly $8 \%$. The model's ability to capture the velocity profile, estimate the jet-induced boundary layer thickness, and calculate the induced jet strength was evaluated using experimental data and results from the selected numerical models. The present model shows the most consistent predictive capability and adequate accuracy for all test cases in comparison to other numerical scenarios. The results show that the proposed model and technique have a bright future in plasma flow control applications.

\section{Methods}

The experimental setup and the plasma actuator investigated in this study are illustrated in Figure 15. The plasma device is comprised of two $0.05 \mathrm{~mm}$ thick aluminum electrodes and six layers of Kapton film as a dielectric. The exposed electrode and embedded electrode have widths of $10 \mathrm{~mm}$ and $30 \mathrm{~mm}$, respectively. In the streamwise direction, the spacing between the upper and lower electrodes is adjusted to zero. Each actuator is $0.4 \mathrm{~m}$ long, and velocity field measurements were performed along the actuator device's centerline. Measurements were obtained using a $1.6 \mathrm{~mm}$ outer diameter glass pitot tube in quiescent air. The probe was directed to measure the streamwise velocity and mounted to a vertical traverse with a resolution of $0.01 \mathrm{~mm}$. Each velocity data point was averaged over a sample measured at $5 \mathrm{kHz}$ for an interval of 10 seconds. The sample population was recorded using a NI-USB5239 data acquisition device connected to a PC. The downstream location of the measurement relative to the trailing edge of the exposed electrode for each actuator is set to be $5 \mathrm{~mm}$ and $12.5 \mathrm{~mm}$ respectively, for two study cases. Each excitation signal was sinusoidal and was delivered using a Rigol DG1011 waveform generator. The output sine wave voltage and frequency range of the power supply were, respectively, 0 to $15 \mathrm{kV}$ and 0 to $15 \mathrm{kHz}$. The steady-state wave was used in all of the experiments.

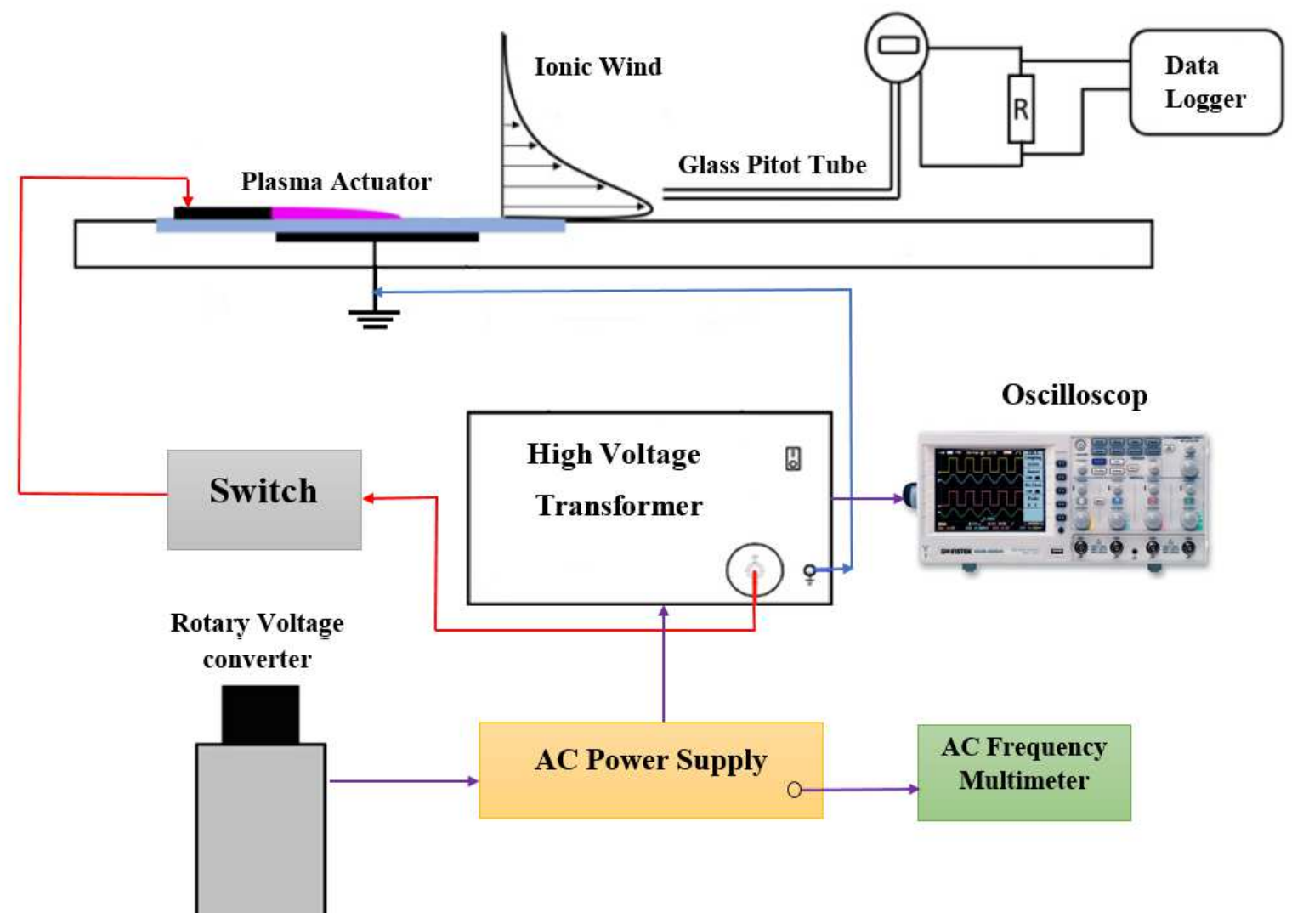

Figure 15. Schematic representation of the experimental setup of the conducted experiment

\section{References}

1. Rodriguez, J. A. et al. Inverse design of plasma metamaterial devices for optical computing. arXiv preprint arXiv:2102.05148 (2021). 
2. Kourtzanidis, K., Pederson, D. M. \& Raja, L. L. Electromagnetic wave energy flow control with a tunable and reconfigurable coupled plasma split-ring resonator metamaterial: A study of basic conditions and configurations. J. Appl. Phys. 119, 204904 (2016).

3. Hamouda, I., Labay, C., Cvelbar, U., Ginebra, M.-P. \& Canal, C. Selectivity of direct plasma treatment and plasmaconditioned media in bone cancer cell lines. Sci. reports 11, 1-12 (2021).

4. Roy, S., Choudhury, B., Johnson, J. \& Schindler-Tyka, A. Application of dielectric barrier discharge for improving food shelf life and reducing spoilage. Sci. Reports 11, 1-9 (2021).

5. Deylami, H. M., Amanifard, N., Dolati, F., Kouhikamali, R. \& Mostajiri, K. Numerical investigation of using various electrode arrangements for amplifying the ehd enhanced heat transfer in a smooth channel. J. Electrost. 71, 656-665 (2013).

6. Kazemi, M., Ghanooni, P., Mani, M. \& Saeedi, M. Drag reduction of 3d bluff body using sdbd plasma actuators. Proc. Inst. Mech. Eng. Part D: J. Automob. Eng. 235, 1461-1480 (2021).

7. Sato, S., Furukawa, H., Komuro, A., Takahashi, M. \& Ohnishi, N. Successively accelerated ionic wind with integrated dielectric-barrier-discharge plasma actuator for low-voltage operation. Sci. reports 9, 1-11 (2019).

8. Portugal, S., Roy, S. \& Lin, J. Functional relationship between material property, applied frequency and ozone generation for surface dielectric barrier discharges in atmospheric air. Sci. reports 7, 1-11 (2017).

9. Pinchuk, M. et al. Role of charge accumulation in guided streamer evolution in helium dbd plasma jets. Sci. reports 11, $1-11$ (2021).

10. Rizzetta, D. P. \& Visbal, M. R. Plasma-based flow-control strategies for transitional highly loaded low-pressure turbines. J. fluids engineering 130 (2008).

11. Thomas, F., Kozlov, A. \& Corke, T. Plasma actuators for landing gear noise reduction. In 11th AIAA/CEAS Aeroacoustics Conference, 3010 (2005).

12. Little, J., Nishihara, M., Adamovich, I. \& Samimy, M. High-lift airfoil trailing edge separation control using a single dielectric barrier discharge plasma actuator. Exp. fluids 48, 521-537 (2010).

13. Whalley, R. D. \& Walsh, J. L. Turbulent jet flow generated downstream of a low temperature dielectric barrier atmospheric pressure plasma device. Sci. reports 6, 1-7 (2016).

14. Labergue, A., Moreau, E., Zouzou, N. \& Touchard, G. Separation control using plasma actuators: application to a free turbulent jet. J. Phys. D: Appl. Phys. 40, 674 (2007).

15. Gibson, B., Arjomandi, M. \& Kelso, R. The response of a flat plate boundary layer to an orthogonally arranged dielectric barrier discharge actuator. J. Phys. D: Appl. Phys. 45, 025202 (2011).

16. Jukes, T. N. \& Choi, K.-S. Long lasting modifications to vortex shedding using a short plasma excitation. Phys. review letters 102, 254501 (2009).

17. Likhanskii, A. V., Shneider, M. N., Macheret, S. O. \& Miles, R. B. Modeling of dielectric barrier discharge plasma actuator in air. J. Appl. Phys. 103, 053305 (2008).

18. Hoskinson, A. R., Hershkowitz, N. \& Ashpis, D. E. Force measurements of single and double barrier dbd plasma actuators in quiescent air. J. Phys. D: Appl. Phys. 41, 245209 (2008).

19. Yoon, J.-S. \& Han, J.-H. Semiempirical thrust model of dielectric barrier plasma actuator for flow control. J. Aerosp. Eng. 28, 04014041 (2015).

20. Amanifard, N., Abdollahzadeh, M., Moayedi, H. \& Pascoa, J. An explicit cfd model for the dbd plasma actuators using wall-jet similarity approach. J. Electrost. 107, 103497 (2020).

21. Lemire, S., Vo, H. D. \& Benner, M. W. Performance improvement of axial compressors and fans with plasma actuation. Int. J. Rotating Mach. 2009 (2009).

22. Mertz, B. E. Refinement, validation, and implementation of lumped circuit element model for single dielectric barrier discharge plasma actuators (University of Notre Dame, 2010).

23. Shyy, W., Jayaraman, B. \& Andersson, A. Modeling of glow discharge-induced fluid dynamics. J. applied physics 92, 6434-6443 (2002).

24. Suzen, Y., Huang, G., Jacob, J. \& Ashpis, D. Numerical simulations of plasma based flow control applications. In 35th AIAA Fluid Dynamics Conference and Exhibit, 4633 (2005). 
25. Abdollahzadeh, M., Pascoa, J. \& Oliveira, P. Modified split-potential model for modeling the effect of dbd plasma actuators in high altitude flow control. Curr. Appl. Phys. 14, 1160-1170 (2014).

26. Suzen, Y. \& Huang, G. Simulations of flow separation control using plasma actuators. In 44th AIAA Aerospace Sciences Meeting and Exhibit, 877 (2006).

27. Enloe, C. L. et al. Mechanisms and responses of a dielectric barrier plasma actuator: Geometric effects. AIAA journal $\mathbf{4 2}$, 595-604 (2004).

28. Enloe, C., McLaughlin, T., Van Dyken, R. \& Fischer, J. Plasma structure in the aerodynamic plasma actuator. In 42nd AIAA Aerospace Sciences Meeting and Exhibit, 844 (2004).

29. Omidi, J. \& Mazaheri, K. Micro-plasma actuator mechanisms in interaction with fluid flow for wind energy applications: Physical parameters. Phys. Fluids 32, 077107 (2020).

30. Omidi, J. \& Mazaheri, K. Differential evolution algorithm for performance optimization of the micro plasma actuator as a microelectromechanical system. Sci. Reports 10, 1-18 (2020).

31. Bouchmal, A. Modeling of dielectric-barrier discharge actuator. Master Sci. Thesis, Delft Univ. Technol. (2011).

32. Ibrahim, I. \& Skote, M. Simulations of the linear plasma synthetic jet actuator utilizing a modified suzen-huang model. Phys. fluids 24, 113602 (2012).

33. Orlov, D. \& Corke, T. Numerical simulation of aerodynamic plasma actuator effects. In 43rd AIAA Aerospace Sciences Meeting and Exhibit, 1083 (2005).

34. Orlov, D., Corke, T. \& Patel, M. Electric circuit model for aerodynamic plasma actuator. In 44th AIAA Aerospace Sciences Meeting and Exhibit, 1206 (2006).

35. Roy, S. \& Gaitonde, D. Radio frequency induced ionized collisional flow model for application at atmospheric pressures. $J$. Appl. Phys. 96, 2476-2481 (2004).

36. Roy, S. \& Gaitonde, D. Modeling surface discharge effects of atmospheric rf on gas flow control. In 43rd AIAA Aerospace Sciences Meeting and Exhibit, 160 (2005).

37. Jayaraman, B. \& Shyy, W. Modeling of dielectric barrier discharge-induced fluid dynamics and heat transfer. Prog. Aerosp. Sci. 44, 139-191 (2008).

38. Hall, K., Jumper, E., Corke, T. \& McLaughlin, T. Potential flow model of a plasma actuator as a lift enhancement device. In 43rd AIAA Aerospace Sciences Meeting and Exhibit, 783 (2005).

39. Weile, D. S. Electromagnetic metamaterials: Physics and engineering explorations (engheta, n. and ziolkowski, rw; 2006)[book review]. IEEE Antennas Propag. Mag. 49, 137-139 (2007).

40. Corke, T. C., Enloe, C. L. \& Wilkinson, S. P. Dielectric barrier discharge plasma actuators for flow control. Annu. review fluid mechanics 42, 505-529 (2010).

41. Kotsonis, M. \& Veldhuis, L. Experimental study on dielectric barrier discharge actuators operating in pulse mode. J. Appl. Phys. 108, 113304 (2010).

42. Palmeiro, D. Modeling of dielectric barrier discharge plasma actuators for flow control simulations. Ph.D. thesis (2011). 